\title{
SOME APPLICATIONS OF MEASUREMENTS OF WHOLE-BODY RADIOACTIVITY IN CLINICAL MEDICINE
}

\author{
D. Hughes, P. R. J. Burch, and A. R. Wilson
}

Interest in the radioactivity of the human body stemmed initially from the use of radium salts medicinally and the ingestion of radium, with tragic consequences, by the early luminizers. The first measurements of body radioactivity were reported by Schlundt, Barker and Flinn (1929) who could estimate in vivo body burdens of radium in the region of 5 to $100 \mu \mathrm{g}$. Instruments with a sensitivity sufficient to detect the natural gamma-ray activity of the body were first developed by Sievert (I95I) and Burch and Spiers (1953). The gamma radiation from the body normally arises from the radioactive isotope, ${ }^{40} \mathrm{~K}$, which is present in all natural potassium with a constant abundance of $0.0118 \%$. These researchers used high-pressure ionization chambers and, with an observation time of two hours, could estimate body potassium with a standard error of 30 to $50 \%$. Stimulated by the needs of radiological protection with the increasing use of atomic energy and radioactive isotopes, the newly developed scintillation detectors were employed in the later 1950's to construct yet higher sensitivity counters which had the further advantage of enabling gamma-rays of differing energies to be distinguished by pulse height selection techniques. These developments resulted in an increase in sensitivity of 50,000 times that of Schlundt's original apparatus and today body potassium can be measured with a standard error of $I$ or $2 \%$ in an observation time of 15 minutes (Spiers, 1962). Clearly the determination of body potassium with this order of ease and accuracy is of great clinical value and practical usefulness. Furthermore, with this extreme sensitivity, tracer studies with radioisotopes can now be conducted over extended periods of time with very low levels of administered activity.

In Leeds, two whole-body counters are in operation which largely complement one another. In the first, shown in Fig. I, three plastic scintillator detectors $\left(20\right.$ in. $\times$ ro in. $\times 6 \frac{1}{2}$ in.) are grouped around a seated subject to give a system of high sensitivity and moderate energy resolution, well suited to body potassium measurements (Burch, Hughes, Iinuma, Overton and Appleby, 1962). In the other, shown in Fig. 2, four sodium iodide crystals (6 in. $\times 4$ in.) are arranged two above and two below a supine subject. It is also possible to locate individual detectors near specific
WHOLE BODY COUNTER IN STEEL ROOM

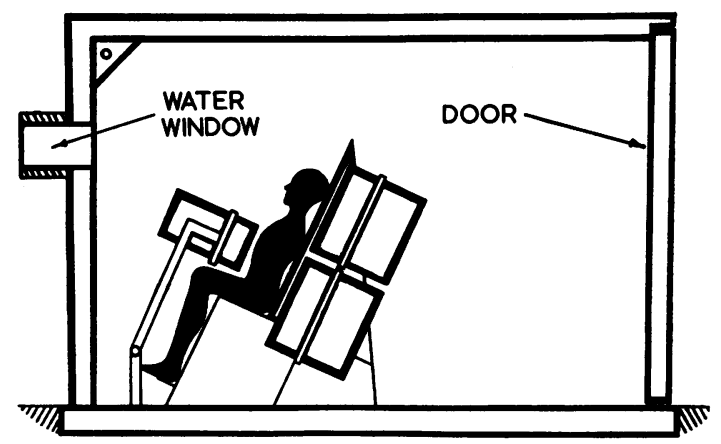

FIG. I.-Plastic scintillator whole-body radioactivity counter in steel room.

sites in the body and thus provide information on the distribution of the radioactivity. Although this second apparatus has a lower sensitivity than the first, it possesses the great advantage of high resolution of different $\gamma$-ray energies and this permits study of several radioisotopes administered simultaneously.

Each of the instruments is housed in a 5 -in. thick steel cubicle lined with up to 2 in. of lead to screen the detectors from natural or other gamma-radiation in the background. The hospital laboratory in which they are situated is shielded from therapeutic and other radiation sources in the hospital. Patients enter a cubicle through a manually operated door (weighing three tons!) and are observed through an 18-in. thick water-filled window. The patient can see out and has two-way speech communication with the operator outside; these facilities minimize possible claustrophobia.

Most of our clinical work has centred so far on the measurement of potassium. The whole-body counter provides a direct index for the study of potassium depletion which otherwise can be only inferred from a knowledge of serum concentration. Also, the technique is more straightforward and rapid than the ${ }^{42} \mathrm{~K}$ dilution method of measuring exchangeable potassium (variously reported as 85 to $98 \%$ of total potassium) and, in general, it does not require the administration of any radioactive material. Our present investigations include studies of potassium in diuretic therapy, preg- 
WHOLE - BOOY MONITER NO. 4.

FIXED - GEOMETRY SIDE ELEVATION.

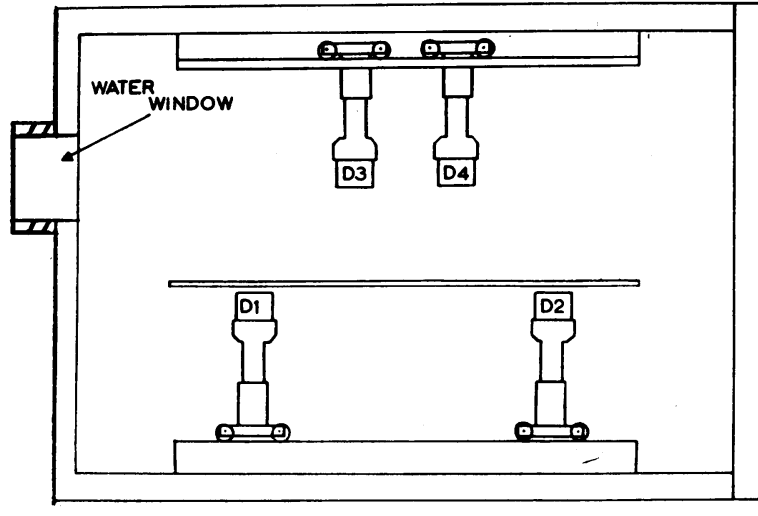

SCANNING - GEOMETRY END ELEVATION.

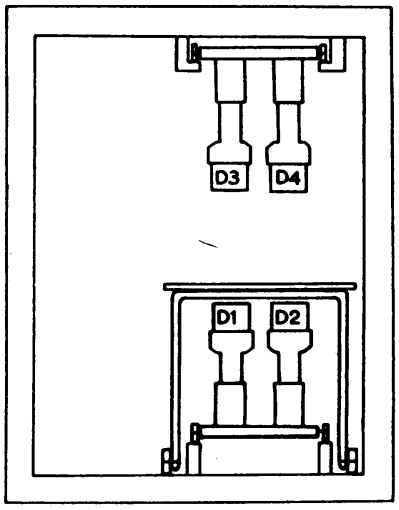

D1, D2,03,804: 6"x4" NaI(TI) CRYSTALS.

STEEL CUBICLE: INTERNAL DIMENSIONS: $10^{\prime} \mathrm{long} 6^{\prime} 6^{\prime \prime}$ high 5 'wide.

FIG. 2.-Sodium iodide crystal whole-body radio-activity counter.

nancy, muscular dystrophy, and various renal disorders. In diuretic therapy, for example, I $\mathrm{g}$. of chlorothiazide was administered daily to 12 normal volunteers. This produced a fall in serum potassium level but no significant change was observed in the whole-body potassium during the four weeks' duration of the investigation.

When a patient is suspected of having a potassium deficiency, it is necessary to compare the measured result with a control value. This value can be obtained in terms of sex, age, and gross weight from data accumulated for normals. We are now investigating, however, the relationship of whole-body potassium to total body water in the hope that this will offer a closer correlation than that of potassium with gross body weight. Conversely, in patients in whom a normal potassium content is considered likely on clinical grounds, a knowledge of the control value of body water in relation to measured body potassium can provide an index for the management of œdema.

In tracer investigations, the whole-body counter measures directly the retention of the administered isotope and so avoids possible errors due to incomplete collections if excreta are assayed. In 을 a study designed to compare the total body counting method with the excretion method, $\bar{z}$ doses of $\mathrm{I} \cdot 5 \mu \mathrm{c}{ }^{47} \mathrm{Ca}$ were administered to seven subjects and, at the end of one week, the activities $\stackrel{\rho}{工}$ in the body, and in the total collected urine and $\vec{\varphi}$ fæces were measured. For six of the subjects summation of the three measurements lay withi the limits of 0.89 to r.ro times the administere dose. Within the limits of accuracy of the measurements, set partly by changes in distribution of the ${ }^{47} \mathrm{Ca}$ in the body, all the activity administered to these patients was accounted for and very little activity could have been lost by any other excretory route, such as in sweat. In the seventh case, the measurements totalled 0.84 times the administered dose, but the relative activities excreted in urine and fæces appeared abnormal.

At present, measurements are being made of the uptake and retention of ${ }^{59} \mathrm{Fe}$ in a patient with obscure iron-loading anæmia. The sodium iodide crystal counter is being used in this case to investigate the retention in liver and spleen in relation to that in other parts of the body.

\section{REFERENCES}

Burch, P. R. J., Hughes, D., Innuma, T. A., Overton, T. R., and Appleby, D. B. (I962): The Construction and Performance of a Three-unit Plastic Scintillator Whole-body Counter. 'Whole-body Counting' (I.A.E.A. Symposium on 'Whole-body Counting', Vienna, r96r), pp. 59-69.

- and SpIERs, F. W. (I953): Measurement of the $\gamma$-Radiation from the Human Body, Nature, $172,519$.

Schlundt, H., Barker, H. H., and Flinn, F. B. (r929): Radium Therapy, Amer. F. Roentgenol ., $21,345$.

SIEVERT, R. M. (1951): Measurement of Gamma-radiation from the Human Body, Arkiv. Fysik., 3, 337.
SPIERS, F. W. (r962): Whole-body Counting; An Introductory Review. 'Whole-body Counting' (I.A.E.A. Symposium on 'Whole-body Counting', Vienna, r96I), p. 3 . 